\title{
Analysis on the wrinkling in continuous roll forming of three-dimensional surface parts
}

\author{
Wei-Xue Hu ${ }^{1, a}$, Qing-Min Chen ${ }^{1, *}$ Xue-Ping Yan ${ }^{1}$ \\ ${ }^{1}$ Roll Forging Research Institute, Jilin University (Nanling Campus), 5988 Renmin Street, \\ Changchun, P.R.China \\ ahuwxjlu@163.com \\ ${ }^{*}$ Corresponding author: chenqm@jlu.edu.cn
}

\begin{abstract}
Keywords: Continuous forming; Rolling; Wrinkling; Numerical simulation; Three-dimensional surface
\end{abstract}

\begin{abstract}
Continuous roll forming is a new sheet metal forming process for three-dimensional surface parts. In the process, the sheet metal is thinned non-uniformly in transverse direction by controlling the distribution of the gap between the upper and lower forming rolls, the longitudinal elongation is different, which makes the sheet metal generate a doubly curved surface parts. When sheet metal forming, the complex stress state produced in the sheet. Since the size in thickness is much smaller than in the transverse and longitudinal directions, the energy required for the deformation of the thin plate is small, it is easy to produce wrinkling problems. In this paper, finite element analysis model is established, wrinkling in the spherical surface and saddle surface are simulation and the experimental results of the wrinkling in saddle surface is presented. The results demonstrate that unreasonable distribution of the roll gap is the main reason for the generation of wave-shaped wrinkling.
\end{abstract}

\section{Introduction}

Continuous manufacturing technology is suitable for producing small-lot or single production as for the low set-up cost. Yoou and Yang developed a method known as an incremental roll forming process which two pairs of lower support rolls and one upper center roll are adopted [1,2]. Since the bending happens at the local contact area just under the upper center roll, and with the rotating upper center roll, the sheet mental is deformed continuously. Shim et al.[2] developed the line array roll set process which was composed of three rows of upper rolls and three rows of lower rolls, and each row of rolls was composed of multiple independent short rolls. Continuous flexible forming process for the fabrication of three-dimensional sheet metal parts was proposed based on the idea of roll-bending using flexible rolls [3,4]. The continuous flexible forming process employs three flexible rolls controlled at multi-point as forming tools, the rotations of the flexible rolls drive the sheet metal feeding and bend the sheet metal in the longitudinal direction and transversal direction simultaneously, and thus a three-dimensional surface formed continuously. To realize rapidly forming of three-dimensional surface part, rolling technique, which is usually used in bulk forming, is applied to sheet metal forming and a new process to manufacture curved surface parts, so-called continuous roll forming has been proposed [5-7]. The process combines rolling technique with the bendable rolls for manufacturing three-dimensional surface part [5], by controlling the roll gap between the upper and lower forming rolls, doubly bending deformation generated. Cai et al. [5,6] theoretically analyzed bending deformation of sheet metal in continuous roll forming process, the bending equations of longitudinal and lateral were set forth, and process design methods were given. Wrinkling is one of the common defects in forming parts. In this paper, finite element analysis model is established, a series of experiments have been carried out. Analysis of the simulation results and the experimental results in the the wrinkling problem, the form and law of the wrinkling are explored. Unreasonable distribution of the roll gap can produce wrinkling, so that the precise control of roll gap is very important. 


\section{Principle of continuous roll forming and wrinkling problems}

As shown in Figure 1a, the continuous roll forming system is composed of an upper bendable and a lower bendable roll. Roll gap distributed non-uniformly in the transversal direction; the sheet metal compressed non-uniformly so that non-uniform elongation in the longitudinal direction takes place. The sheet mental bent in the transversal direction and non-uniform elongation in the longitudinal direction, a three-dimensional surface part formed finally. With the rotations of the upper and lower forming rolls, the sheet mental is continuously shaped into a doubly curved surface. Rolling equipment shown in Figure $1 b$.

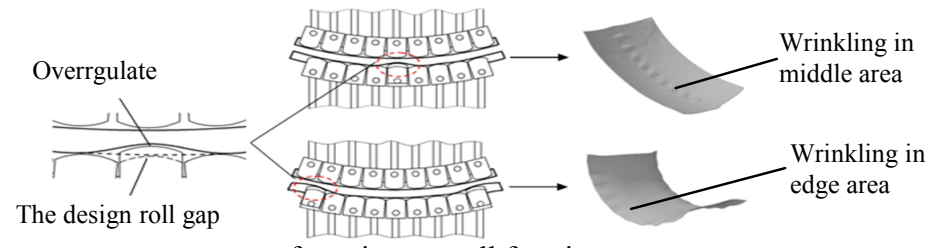

a. principle of continuous roll forming

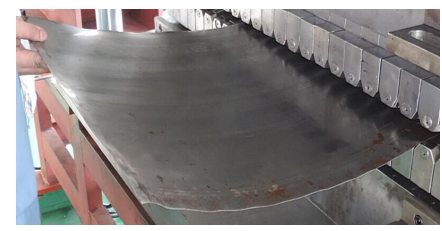

b. rolling equipment

Fig. 1 Principle of continuous roll forming and rolling equipment

If the roll gap is relatively small in the middle and large on the two edges, a surface with positive Gaussian curvature produced. If the roll gap is relatively large in the middle and small on the two edges, a surface with negative Gaussian curvature produced.

Wrinkling is a typical quality defects in the rolling process, it is a defective form of sheet material produced by compression buckling. The rational design of the roll gap is the basis of the continuous roll forming, roll seam design with an error; it may lead to wrinkling problems. Shown in Figure 1 a, when the difference of the amount of compression of the roll gap between the local and surrounding area is large, and it is easy to lead to wrinkling. Excessive adjustment in both sides of the roll gap will lead to wrinkling on both sides. Excessive adjustment in iintermediate of the roll gap will lead to wrinkling in the middle area. In Figure 1a, a roll forming parts with wrinkling is given. The wrinkling area in Negative Gaussian curvature and positive Gaussian curvature surfaces are different. In negative Gaussian curvature surface, sheet metal thinning in the middle with a small amount as well as a large amount on both sides. Thinning increasing on both sides, sheet is prone to wrinkling on both sides. In positive Gaussian curvature surface, sheet thinning in the middle with a small amount and a large amount on both sides. The amount of thinning increasing in the middle position, sheet wrinkling generated in the middle.

\section{Numerical simulation analysis of wrinkling problem}

Irrational distribution of the roll gap will lead to sheet wrinkling. Under different offset of the roll gap, wrinkling in different positions analyzed through the following numerical simulation. Figure 2a shown the offset position of the roll gap, D1, D2, D3, D4, D5 is the offset in different positions. When offset of the roll gap occurs in both sides, D1 is the maximum offset and the value decreases from D1 to D5, if the offset occurs in the middle of the roll gap, the maximum offset is D6, and the offset decreases from D6 to D9.Figure $2 b$ is the corresponding finite element model.

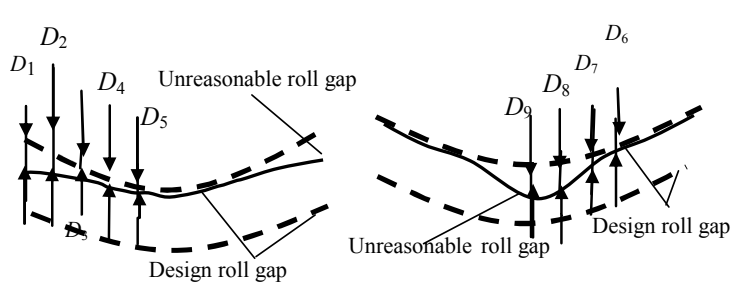

a design roll gap
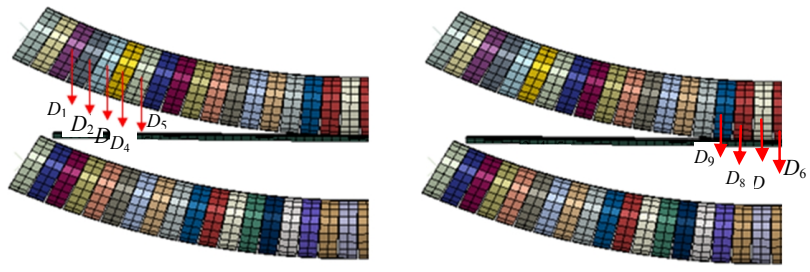

b. finite element model

Fig 2 Deviation of the roll gap 
In order to understand the causes of wrinkling, the deformations of sheet metal in different roll gap offset analyzed. When wrinkling occurs in both sides, in figure 3a, the changing of the curvature radius of the longitudinal with maximum offset D1 are given. With the increasing of the maximum offset D1, the longitudinal curvature radius of the formed parts decrease from $290 \mathrm{~mm}$ to $210 \mathrm{~mm}$. As the maximum offset D1 reaches $0.075 \mathrm{~mm}$, the longitudinal curvature radius increase with the increase of the maximum offset, if the value of maximum offset D1 continues to increase, there will be a wrinkling defect, Dmax $=0.075 \mathrm{~mm}$ is the maximum offset that does not produce wrinkling. As shown in Figure $3 b$, as wrinkling in the middle area, the variation of the longitudinal curvature radius under different offset has a similar tendency.
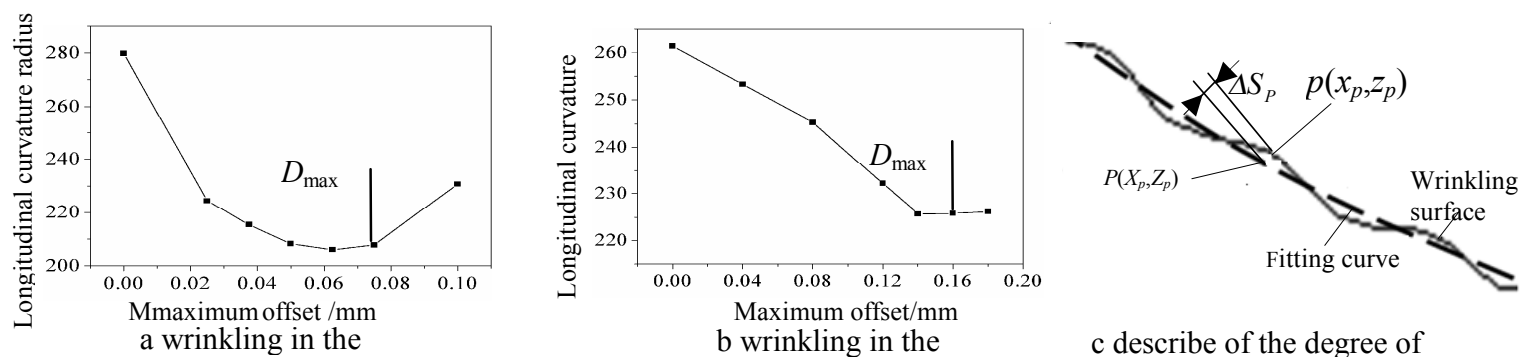

Fig 3 two kinds of wrinkling and describe of the degree of wrinkling

Figure $3 \mathrm{c}$ is the schematic view of the cross-section line and the fitting surface in the center of the local area. $\Delta S_{P}$ is the wrinkle height, the maximum value of $\Delta S_{P}$ indicates the degree of wrinkling, that is $\Delta S_{\max }$.

Tab. 1 Offset on different areas $(\mathrm{mm})$

a. Offset in middle areas $(\mathrm{mm})$

\begin{tabular}{ccccc}
\hline & D6 & D7 & D8 & D9 \\
\hline C5 & 0 & 0 & 0 & 0 \\
C6 & 0.2 & 0.15 & 0.1 & 0.05 \\
C7 & 0.22 & 0.17 & 0.1 & 0.05 \\
C8 & 0.25 & 0.2 & 0.1 & 0.05 \\
\hline
\end{tabular}

b. Offset in both sides (mm)

\begin{tabular}{cccccc}
\hline & D1 & D2 & D3 & D4 & D5 \\
\hline C1 & 0 & 0 & 0 & 0 & 0 \\
C2 & 0.10 & 0.08 & 0.06 & 0.04 & 0.02 \\
C3 & 0.15 & 0.12 & 0.09 & 0.06 & 0.03 \\
C4 & 0.20 & 0.16 & 0.12 & 0.08 & 0.04 \\
\hline
\end{tabular}

In order to analysis the influence of roll gap offset on wrinkling in the middle area, four different groups of roll gap used in the numerical simulation. C5 roller seam distribution strictly satisfy the forming surface with positive Gaussian curvature, C6, C7, C8 roll gap distribution are shown in table 1a In order to analysis the influence of roll gap offset on wrinkling in both sides, four different groups of roll gap distribution are used in the numerical simulation. $\mathrm{C} 1$ roller seam distribution strictly satisfy the forming surface with negative Gaussian curvature, C2, C3, C4 roll gap distribution as shown in table $1 b$.

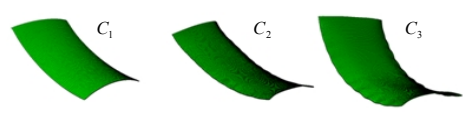

(1) wrinkling in both sides

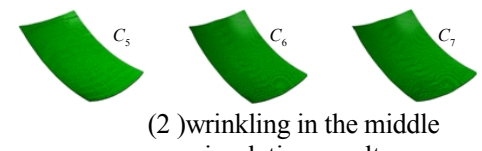
a simulation results
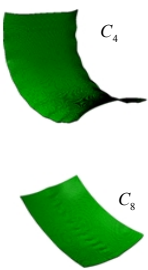

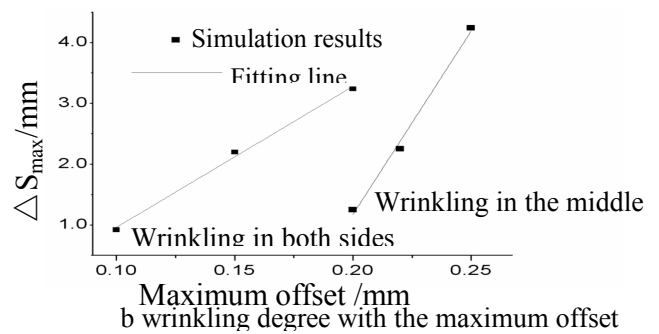

Fig 4 Results of the numerical simulation

Figure $4 \mathrm{a}(1)$ and $\mathrm{b}$ are the results of the numerical simulation of wrinkling in both sides. With the increasing of the maximum offset, the degree of wrinkling becomes more and more obvious. When the maximum offset D1 is $0 \mathrm{~mm}$, the forming quality is good, when the maximum offset is $0.1 \mathrm{~mm}$, the forming parts appear wrinkling, $\Delta S_{\max }=0.92 \mathrm{~mm}$, with the increasing of the maximum offset, wrinkling 
become more and more obvious, when the maximum offset reaches $0.2 \mathrm{~mm}$, wrinkling is very serious, $\Delta S_{\max }=3.24 \mathrm{~mm}$. Figure $4 \mathrm{a}(2)$ and $\mathrm{c}$ are the results of the numerical simulation of wrinkling in the middle. When the maximum offset D6 $=0$, the forming parts has a good quality, wrinkling appears when D6 $=0.2 \mathrm{~mm}$, and the maximum height of wrinkling $\Delta S_{\max }=1.25 \mathrm{~mm}$. When D6 $=0.25 \mathrm{~mm}$, $\Delta S_{\max }=4.24 \mathrm{~mm}$.

\section{Experimental research on wrinkling}

In order to verify the correctness of the simulation analysis of the rolling wrinkling phenomenon, a series of experiments have done. As shown in Fig 5a, four saddle surfaces present, from a1 to a4, the roll gap offset decreases. The wrinkling in a1 is serious, $\Delta S_{\max }=3.96 \mathrm{~mm}$, with the decrease of the roll gap offset, wrinkling degree decreased gradually. After four times adjustment, roll gap offset near zero and wrinkling disappeared, and the tendency shown in Fig 5b.

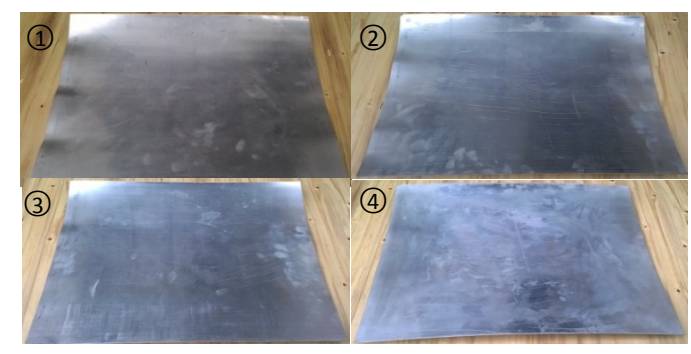

a experimental parts with wrinkling

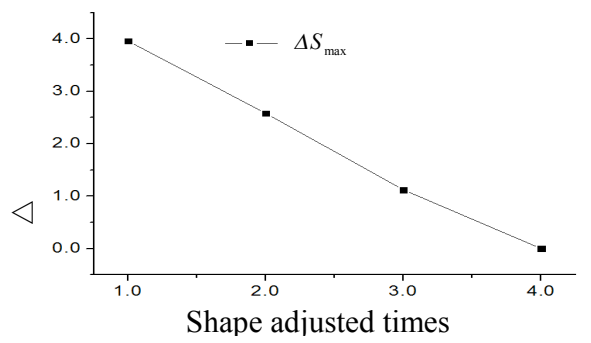

$\mathrm{b}$ the change of wrinkling degree with roll gap offset

Fig 5 exprimental results

\section{Conclusions}

(1)The mechanism of wrinkling in the rolling parts is studied. According to the characteristics of surface forming in continuous rolling, the roll gap distribution corresponds to the wrinkling zone is analyzed.

(2)Positions and forms of wrinkling have analyzed by numerical simulation. The influence factor of the wrinkling degree also studied.

(3)The simulation analysis of the causes of wrinkling defects are verified by experiment.

\section{References}

[1] S. J. Yoon, D.Y.Yang. An incremental roll forming process for manufacturing doubly curved sheets from general quadrilateral sheet blanks with enhanced process features. CIRP Ann Manufacturing Technology, Vol.54 (2005), p 221-224

[2] S. J. Yoon, D.Y.Yang. Development of a Highly Flexible Incremental Roll Forming Process for the Manufacture of a Doubly Curved Sheet Metal. CIRP Annals - Manufacturing Technology, Vol.52 (2003), p.201

[3] Z.Y. Cai, M.Z. Li and Y. W. Lan. Three-dimensional sheet metal continuous forming process based on flexible roll bending: principle and experiments. Journal of Materials Processing Technology, Vol. 212 (2012), p.120

[4] Z. Sui, Z.Y. Cai, Y.W. Lan. Simulation and software design of continuous flexible roll bending process for three dimensional surface parts, Materials \& Design, Vol.54 (2014), p.498

[5] Z.Y. Cai, M.Z. Li. Principle and theoretical analysis of continuous roll forming for three- dimensional surface parts. Science China Technological Sciences, Vol.56 (2013), p.351

[6] Z.Y. Cai, M. Wang, M.Z. Li. Study on the continuous roll forming process of swept surface sheet metal part. Journal of Materials Processing Technology, Vol.214 (2014), p.1820

[7] Z.Y. Cai, D.B. Guan, M. Wang. A novel continuous roll forming process of sheet metal based on bended rolls, International Journal of Advanced Manufacturing Technology, Vol.73 (2014), p. 1807 